\title{
Performance of broiler chickens at pre starter and starter phases using diets with different metabolizable energy values of ingredients, at different ages
}

\author{
Desempenho de pintos de corte nas fases pré-inicial e inicial, utilizando dietas com \\ diferentes valores de energia metabolizável dos ingredientes, em diferentes idades
}

\author{
José Luiz Schneiders $^{\mathrm{I}}$ Ricardo Vianna Nunes $^{\mathrm{I}}$ Thaís Lorana Savoldi ${ }^{\mathrm{I}}$ Liliane Borsatti $^{\mathrm{I}}$ \\ Rodrigo André Schöne ${ }^{I}$ Rafael Frank $^{I}$ Douglas Fernando Bayerle ${ }^{I}$ Idiana Mara da Silva
}

ABSTRACT

The experiment was carried out to evaluate the performance of broilers in 1-7 and 8-21 days of age, using metabolizable energy values of corn, soybean meal and soybean oil previously determined for each phase in another metabolism trial. Two trails were conducted, divided into phases according to ages: pre-starter (1-7 days) and starter (8-21 days). At each trial were used 924 broiler chicken randomly distributed into 42 experimental units in a completely randomized design with six treatments and seven replications. Treatments consisted of five diets using the values of apparent metabolizable energy corrected for nitrogen balance of corn, soybean meal and soybean oil determined in different metabolism trials, and one diet with the metabolizable energy values of these same ingredients, published on the literature. At the end of each stage, the animals and feed leftover were weighted to determine feed intake, weight gain and feed conversion ratio. Diets with higher soybean oil inclusion, showed superior performance at different stages. At pre-starter only feed conversion ratio was significant $(P<0.05)$. In the initial phase, there was a significant difference $(P<0.05)$ for feed conversion ratio, daily and average weight gain.

Key words: energetic coefficients, feed conversion ratio, feed intake, weight gain.

\section{RESUMO}

$O$ experimento foi realizado para avaliar o desempenho de frangos de corte nas idades de 1-7 e 8-21 dias de idade, utilizando valores de energia metabolizável do milho, farelo de soja e óleo de soja determinados anteriormente em outro ensaio de metabolismo. Dois estudos foram realizados, divididos em fases de acordo com as idades: pré-inicial (1-7 dias) e inicial (8-21 dias). Em cada estudo, foram utilizados 924 pintos de corte distribuidos aleatoriamente em 42 unidades experimentais, em um delineamento inteiramente casualizado consistindo de cinco dietas utilizando os valores de energia metabolizável aparente corrigida para o balanço de nitrogênio do milho, farelo de soja, e óleo de soja, determinados nos ensaios de metabolismo, e uma dieta com os valores de energia metabolizável dos mesmos alimentos publicados na literatura. No final de cada fase, os animais e as sobras de ração foram pesadas para determinar o consumo de alimento, ganho de peso e conversão alimentar. As dietas com maiores inclusões de óleo de soja mostraram desempenho superior nas diferentes idades. Na fase pré-inicial, apenas a conversão alimentar foi significativa. Na fase inicial, houve diferença significativa para a conversão alimentar e ganho de peso médio e diário.

Palavras-chave: coeficientes energéticos, consumo de ração, conversão alimentar, ganho de peso.

\section{INTRODUCTION}

As consequence of faster growing, broiler chickens were slaughter in earlier phases, which required less feed to reach the final weight and allowed production costs reduction. Genetic programs for growing has led to increased feed intake, and consequently to greater fat deposition due to ad libitum access to food, as seen in the broilers production. In addition, modern strains of fast-growing broilers have great potential to develop abnormalities, leading to death, leg and carcass deformities, also reproductive abnormalities, increasing the number of non-conformity hatching eggs and must be; therefore adequate nutrition to new poultry requirements (VIANA et al., 2000).

Broiler performance is intimately related to dietary energy levels, because energy content is

\footnotetext{
'Departamento de Zootecnia, Centro de Ciências Agrárias, Universidade Estadual do Oeste do Paraná (UNIOESTE), 85960-000, Marechal Cândido Rondon, PR, Brasil. E-mail: ze.luiz@zootecnista.com.br. "Corresponding author. Received 02.19.15 Approved 04.28.16 Returned by the author 07.20.16 CR-2015-0224.R5
} 
limiting factor for ingestion, being used in different metabolic processes involving maintenance to maximum productive potential expression. Thus, the accuracy on metabolizable energy (ME) determination is important to reach optimal performance of poultry, since it may reflect in weight gain and feed conversion ratio improvement (DALE \& FULLER, 1982).

Dietary energy contents substantially interfere in broilers performance. According to BASTOS et al. (1998), ALBUQUERQUE et al. (2000), LUCHESI (2000), ROSA et al. (2000), SILVA et al. (2000), MOREIRA et al. (2004) and WATANABE et al. (2001) high energy diets promotes better weight gain and feed conversion ratio, but also increased the abdominal fat.

Thus, the aim of this study was to evaluate the performance (feed intake, feed conversion ratio and weight gain) of broiler chicks at two different stages ( 1 to 7 and 8 to 21 days old), fed with diets with metabolizable energy values determined with five different ages broilers.

\section{MATERIALS AND METHODS}

To evaluate the broilers' performance, were realized two different trials. The diet of each trial was formulated to meet the nutritional requirements of animals divided into phases according to ages: pre-starter (1-7 days) and starter (8-21 days) In both phases, were used 924 broiler chicks. Poultry were randomly distributed into 42 experimental units (EU), according to average weight.

In both trials male broilers chicks were provided by Cobb 500 strain, from broiler breeders with 40 to 50 weeks of age. The poultry were acquired in commercial hatcheries and vaccinated against Marek, Gumboro, Bouba Avian, and Infectious Bronchitis disease.

The chicks used to perform the pre-starter trial ( 1 to 7 days) were not used in the next phase. The animals used in period 8-21 days of age were reared in concrete floors covered with wood shavings litter, receiving starter diet and water ad libitum. At 8th day of age were transferred to boxes (EU) for carry on the trial. Broilers received food and water ad libitum and when necessary the heat system was used, a 250 watts infrared lamps in each EU. Lighting program used was of 24 hours (natural and artificial). Pinnus wood shaving was used as litter.

The experimental design been completely randomized with six treatments and seven replications. The treatments consisted of 5 diets using the apparent digestibility coefficients corrected for nitrogen balance (ADCn) values for corn, soybean meal and soybean oil determined in previous trial conducted by SCHNEIDERS et al. (2011), and a diet formulated with metabolizable energy values from the same feed ingredients published by ROSTAGNO et al. (2005). Thus, treatments were divided as follows:

Pre - energy values determined pre ( 1 to 8 days of age); Start - energy values determined at starter stage (11 to 18 days of age); GroI - energy values determined on phase grol (21 to 28 days of age); GroII - energy values determined on phase gro2 (31 to 38 days of age); Final - energy valuex determined on phase fin (41 to 48 days of age); and Tab - tabulated energy values from ROSTAGNO et al. (2005);

Experimental diets (Tables 1 and 2) were formulated to be isocaloric and isonutritives, meeting the nutritional requirements for fast-growing male broilers chicks for each of the two studied phases (ROSTAGNO et al., 2005). For experimental diet formulation, it was used chemical composition of feeds tabled by ROSTAGNO et al. (2005), exception for digestible amino acids and AMEn (apparent metabolizable energy corrected for nitrogen balance) content of corn, soybean meal and soybean oil. These ingredients were sent to COMCAP/UEM for GE (gross energy) determination, after then, used for ADCn determination in a digestibility trial conducted by SCHNEIDERS et al. (2011). Corn and soybean meal aminograms were determined via NIRS and the amino acids meet the nutritional requirements for broilers published by ROSTAGNO et al. (2005) for each studied stage.

At the end of each phase the animals and feed leftovers were weighed to determine feed intake (FI), average weight gain (GAIN), daily weight gain (ADG) and feed conversion ratio (FCR). Smaller broiler chickens and mortality were taken dailys, being the feed availability adjusted according to animal number in each experimental unit (SAKOMURA \& ROSTAGNO, 2007). At each stage it was used two-thermo hygrometer randomly distributed in two boxes, with the purpose of recording the maximum and minimum temperature as well as air relative humidity inside the shed.

Performance results for two phases were analyzed on Statistical Analysis System - SAEG software (UNIVERSIDADE FEDERAL DE VIÇOSA - UFV, 1999), performing analysis of joint variance and within each period SNK test was used at $5 \%$ probability to investigate the effects of different feed ingredients energy values, determined with different poultry age on broiler chicks performance. 
Table 1 - Chemical and centesimal composition of foods given on the pre starter phase (1 to 7 days).

\begin{tabular}{|c|c|c|c|c|c|c|}
\hline Corn (3955kcal CE Kg $\left.{ }^{-1}\right)$ & 44.614 & 49.953 & 48.412 & 50.270 & 49.517 & 54.030 \\
\hline Soybean meal (4.199kcal CE Kg $\left.{ }^{-1}\right)$ & 40.090 & 39.160 & 39.430 & 39.110 & 39.240 & 38.450 \\
\hline Soybean oil (9399kcal CE Kg $\left.{ }^{-1}\right)$ & 8.290 & 3.873 & 5.146 & 3.605 & 4.229 & 0.500 \\
\hline Dicalcium Fosfate & 1.963 & 1.949 & 1.953 & 1.948 & 1.950 & 1.938 \\
\hline Limestone & 0.915 & 0.926 & 0.923 & 0.927 & 0.925 & 0.934 \\
\hline Salt & 0.522 & 0.519 & 0.520 & 0.519 & 0.520 & 0.518 \\
\hline DL-Methionine & 0.387 & 0.380 & 0.382 & 0.380 & 0.381 & 0.375 \\
\hline L-Lysine $\mathrm{HCl}$ & 0.314 & 0.330 & 0.325 & 0.331 & 0.328 & 0.341 \\
\hline L-Threonine & 0.162 & 0.163 & 0.163 & 0.163 & 0.163 & 0.164 \\
\hline L-Valine & 0.082 & 0.082 & 0.082 & 0.082 & 0.082 & 0.082 \\
\hline L-Isoleucine & 0.011 & 0.015 & 0.014 & 0.015 & 0.015 & 0.018 \\
\hline Mineral/vitamin premix ${ }^{1}$ & 0.400 & 0.400 & 0.400 & 0.400 & 0.400 & 0.400 \\
\hline Choline Chloride $60 \%$ & 0.060 & 0.060 & 0.060 & 0.060 & 0.060 & 0.060 \\
\hline Antioxidant $^{2}$ & 0.020 & 0.020 & 0.020 & 0.020 & 0.020 & 0.020 \\
\hline Inert $^{3}$ & 2.170 & 2.170 & 2.170 & 2.170 & 2.170 & 2.170 \\
\hline TOTAL & 100.000 & 100.000 & 100.000 & 100.000 & 100.000 & 100.000 \\
\hline Poultry metabol energy $\left(\mathrm{kcal} \mathrm{kg}^{-1}\right)$ & 2,959 & $\begin{array}{l}\text { Chemical } \\
2,960\end{array}$ & $\begin{array}{l}\text { sition--- } \\
2,960\end{array}$ & 2,960 & 2,960 & 2,961 \\
\hline ADCn corn $(\%)$ & 70.600 & 74.800 & 76.000 & 78.600 & 79.400 & NA \\
\hline ADCn soybean meal (\%) & 46.200 & 54.000 & 49.800 & 48.000 & 47.800 & NA \\
\hline ADCn soybean oil (\%) & 85.400 & 94.800 & 91.800 & 92.200 & 98.600 & NA \\
\hline Crude protein $(\%)$ & 22.990 & 22.990 & 23.000 & 23.000 & 23.000 & 22.990 \\
\hline Calcium (\%) & 0.942 & 0.942 & 0.942 & 0.942 & 0.942 & 0.942 \\
\hline Phosphorous Available (\%) & 0.471 & 0.471 & 0.471 & 0.471 & 0.471 & 0.471 \\
\hline Sodium (\%) & 0.224 & 0.224 & 0.224 & 0.224 & 0.224 & 0.224 \\
\hline Potassium (\%) & 0.859 & 0.857 & 0.857 & 0.857 & 0.857 & 0.855 \\
\hline Methionine dig. poultry (\%) & 0.677 & 0.673 & 0.674 & 0.673 & 0.674 & 0.671 \\
\hline Met.+cist. dig. poultry (\%) & 0.968 & 0.968 & 0.968 & 0.968 & 0.968 & 0.968 \\
\hline Lysine dig. poultry (\%) & 1.363 & 1.363 & 1.363 & 1.363 & 1.363 & 1.363 \\
\hline Isoleucine dig. poultry ( $\%)$ & 0.886 & 0.886 & 0.886 & 0.886 & 0.886 & 0.886 \\
\hline Leucine dig. poultry (\%) & 1.685 & 1.704 & 1.699 & 1.706 & 1.703 & 1.719 \\
\hline Valine dig. poultry (\%) & 1.022 & 1.022 & 1.022 & 1.022 & 1.022 & 1.022 \\
\hline Threonine dig. poultry (\%) & 0.886 & 0.886 & 0.886 & 0.886 & 0.886 & 0.886 \\
\hline Tryptophan dig. poultry (\%) & 0.243 & 0.241 & 0.241 & 0.241 & 0.241 & 0.239 \\
\hline Arginine dig. poultry (\%) & 1.441 & 1.432 & 1.434 & 1.431 & 1.432 & 1.424 \\
\hline Phenylalanine dig. poultry $(\%)$ & 1.016 & 1.016 & 1.016 & 1.016 & 1.016 & 1.016 \\
\hline Histidine dig. Poultry (\%) & 0.564 & 0.565 & 0.565 & 0.565 & 0.565 & 0.565 \\
\hline
\end{tabular}

${ }^{1}$ Starter premix $\left(4 \mathrm{~kg} \mathrm{~T}^{-1}\right)$ : vit A. - 2,500,000UI; vit. D - 625,000UI; vit. E - 6,250UI; vit. K3 - 500mg; vit. B1 - 625mg; vit B2 - 1,625mg; vit. B6 - 875mg; vit. B12 - 4,500mg; Pantothenic acid - 3,750mg; Niacin - 10.50g; Folic acid - 300mg; Biotin - 20mg; Choline - 63.53g; Manganese - 18.74g, Zinc - 17.50g; Organic zinc - 10g; Iron - 11.16g; Copper - 1,996.39mg; Iodine - 187.50mg; Selenium - 75mg; Organic selenium - 25mg; Narazin $-12.50 \mathrm{~g}$; Nicarbazin $-12.50 \mathrm{~g}$; Enramicin $-2,500 \mathrm{mg}$.

${ }^{2}$ BHT.

${ }^{3}$ Sand.

\section{RESULTS AND DISCUSSION}

No significant effect $(\mathrm{P}>0.05)$ was observed on FI, GAIN and ADG; however, significant effect $(\mathrm{P}<0.05)$ was observed for FCR in pre-starter phase (Table 3). Poultry fed diets formulated with metabolizable energy values determined with chicks at 1-8 days old, showed better FCR, showing the importance of formulating diets with energy values determination at pre-start phase, because chicks of 1-7 days of age showed less ability to metabolization, digestion and absorption of nutrients and hence energy. In a trial conducted by LARA et al. (2008), who investigate different dietary energy levels on performance of male and female broiler chicks, founded that higher energy levels provides better FCR both males and females. In the present assay, diets were formulated to be isocaloric, with no differences 
Table 2 - Chemical and centesimal composition of the foods given on the starter phase ( 8 to 22 days).

\begin{tabular}{|c|c|c|c|c|c|c|}
\hline Component & pre & start & gro1 & gro2 & final & tab \\
\hline Corn $\left(3955 \mathrm{kcal} \mathrm{CE} \mathrm{kg}^{-1}\right)$ & 50.474 & 55.466 & 54.232 & 56.039 & 55.558 & 59.763 \\
\hline Soybean meal (4.199kcal CE kg $\left.{ }^{-1}\right)$ & 33.930 & 33.250 & 33.420 & 33.170 & 33.240 & 32.670 \\
\hline Soybean oil (9399kcal CE kg-1) & 8.950 & 4.652 & 5.714 & 4.160 & 4.572 & 1.851 \\
\hline Dicalcium Fosfate & 1.879 & 1.864 & 1.867 & 1.862 & 1.863 & 0.949 \\
\hline Limestone & 0.891 & 0.901 & 0.898 & 0.902 & 0.901 & 0.909 \\
\hline Salt & 0.507 & 0.504 & 0.505 & 0.504 & 0.504 & 0.503 \\
\hline DL-Methionine & 0.312 & 0.304 & 0.306 & 0.303 & 0.303 & 0.296 \\
\hline L-Lysine $\mathrm{HCl}$ & 0.276 & 0.284 & 0.282 & 0.285 & 0.284 & 0.290 \\
\hline L-Threonine & 0.129 & 0.127 & 0.127 & 0.127 & 0.127 & 0.125 \\
\hline L-Valine & 0.052 & 0.048 & 0.049 & 0.048 & 0.048 & 0.044 \\
\hline L-Isoleucine & 0.000 & 0.000 & 0.000 & 0.000 & 0.000 & 0.000 \\
\hline Mineral/vitamin premix ${ }^{1}$ & 0.400 & 0.400 & 0.400 & 0.400 & 0.400 & 0.400 \\
\hline Choline Chloride $60 \%$ & 0.060 & 0.060 & 0.060 & 0.060 & 0.060 & 0.060 \\
\hline Antioxidant $^{2}$ & 0.020 & 0.020 & 0.020 & 0.020 & 0.020 & 0.020 \\
\hline Inert $^{3}$ & 2.120 & 2.120 & 2.120 & 2.120 & 2.120 & 2.120 \\
\hline TOTAL & 100.000 & 100.000 & 100.000 & 100.000 & 100.000 & 100.000 \\
\hline \multicolumn{7}{|c|}{--Chemical composition--- } \\
\hline ADCn corn $(\%)$ & 70.600 & 74.800 & 76.000 & 78.600 & 79.400 & NA \\
\hline ADCn soybean meal (\%) & 46.200 & 54.000 & 49.800 & 48.000 & 47.800 & NA \\
\hline ADCn soybean oil (\%) & 85.400 & 94.800 & 91.800 & 92.200 & 98.600 & NA \\
\hline Crude protein $(\%)$ & 20.446 & 20.521 & 20.523 & 20.529 & 20.523 & 20.586 \\
\hline Calcium (\%) & 0.899 & 0.899 & 0.899 & 0.899 & 0.899 & 0.899 \\
\hline Phosphorous Available (\%) & 0.449 & 0.449 & 0.449 & 0.449 & 0.449 & 0.449 \\
\hline Sodium (\%) & 0.218 & 0.218 & 0.218 & 0.218 & 0.218 & 0.218 \\
\hline Potassium (\%) & 0.762 & 0.764 & 0.763 & 0.764 & 0.764 & 0.765 \\
\hline Methionine dig. poultry (\%) & 0.677 & 0.673 & 0.674 & 0.673 & 0.674 & 0.671 \\
\hline Met.+cist. dig. poultry (\%) & 0.844 & 0.844 & 0.844 & 0.844 & 0.844 & 0.844 \\
\hline Lysine dig. poultry (\%) & 1.189 & 1.189 & 1.189 & 1.189 & 1.189 & 1.189 \\
\hline Isoleucine dig. poultry (\%) & 0.773 & 0.773 & 0.773 & 0.773 & 0.773 & 0.773 \\
\hline Leucine dig. poultry (\%) & 1.543 & 1.567 & 1.561 & 1.570 & 1.568 & 1.588 \\
\hline Valine dig. poultry (\%) & 0.892 & 0.892 & 0.892 & 0.892 & 0.892 & 0.892 \\
\hline Threonine dig. poultry (\%) & 0.773 & 0.773 & 0.773 & 0.773 & 0.773 & 0.773 \\
\hline Tryptophan dig. poultry (\%) & 0.212 & 0.211 & 0.211 & 0.211 & 0.211 & 0.210 \\
\hline Arginine dig. poultry (\%) & 1.267 & 1.264 & 1.265 & 1.264 & 1.264 & 1.262 \\
\hline Phenylalanine dig. poultry $(\%)$ & 0.907 & 0.911 & 0.910 & 0.911 & 0.911 & 0.914 \\
\hline Histidine dig. Poultry (\%) & 0.507 & 0.511 & 0.510 & 0.512 & 0.512 & 0.515 \\
\hline
\end{tabular}

${ }^{1}$ Starter premix $\left(4 \mathrm{~kg} \mathrm{~T}^{-1}\right)$ : vit A. - 2,500,000UI; vit. D - 625,000UI; vit. E - 6,250UI; vit. K3 - 500mg; vit. B1 - 625mg; vit B2 - 1,625mg; vit. B6 - 875mg; vit. B12 - 4,500mg; Pantothenic acid - 3,750mg; Niacin - 10.50g; Folic acid - 300mg; Biotin - 20mg; Choline - 63.53g; Manganese - 18.74g. Zinc - 17.50g; Organic zinc - 10g; Iron - 11.16 g; Copper - 1,996.39mg; Iodine - 187.50mg; Selenium - 75mg; Organic selenium - 25mg; Narazin - 12.50g; Nicarbazin - 12.50g; Enramicin - 2,500mg.

${ }^{2}$ BHT Sand.

in energy levels used among experimental diets. However, different apparent digestibility coefficients corrected for nitrogen balance of corn, soybean meal and soybean oil were used, according to age, in diet formulation leading to a high soybean oil inclusion on EVP pre diet, thus resulting in better FCR.

In order to evaluate the effect of different metabolizable energy and crude protein levels on broilers performance at 0-8 weeks old, HANUSHI et al. (2012) conducted a assay in a $3 \times 3$ factorial arrangement $\left(2,400 ; 2,600\right.$ and $2,800 \mathrm{kcal} \mathrm{kg}^{-1} \times 16$, 18 and $20 \% \mathrm{CP}$ ). There was a difference for energy levels on parameters weight gain, FI and FCR. Animals fed diets with $2,400 \mathrm{kcal} \mathrm{kg}^{-1}$ energy levels had significantly lower performance compared to animals fed with higher energy levels. The authors 
Table 3 - Performance values determined with broiler chicken at pre starter phase (1 to 7 days).

\begin{tabular}{lllllll}
\hline Characteristic & pre & start & gro1 & gro2 & final & tab \\
\hline $\mathrm{BW}^{1}\left(\mathrm{~g} \mathrm{bird}^{-1}\right)$ & 46.490 & 46.470 & 46.160 & 46.450 & 46.760 & 47.050 \\
$\mathrm{FI}^{2}\left(\mathrm{~g} \mathrm{bird}^{-1}\right)$ & 153.570 & 156.920 & 160.800 & 167.140 & 163.120 & 167.670 \\
$\mathrm{FCR}^{3}\left(\mathrm{~g} \mathrm{~g}^{-1}\right)$ & $0.858^{\mathrm{a}}$ & $0.934^{\mathrm{b}}$ & $0.921^{\mathrm{b}}$ & $0.951^{\mathrm{b}}$ & $0.940^{\mathrm{b}}$ & 0.114 \\
$\mathrm{GAIN}^{4}\left(\mathrm{~g} \mathrm{bird}^{-1}\right)$ & 132.440 & 121.710 & 128.490 & 129.200 & 127.080 & 127.900 \\
$\mathrm{ADG}^{5}\left(\mathrm{~g} \mathrm{bird}^{-1} \mathrm{day}^{-1}\right)$ & 18.920 & 17.390 & 18.360 & 18.460 & 18.150 & 18.013 \\
\hline
\end{tabular}

${ }^{*}$ Means followed by the same letters in the same row. Do not differ by SNK test at $5 \%$.

${ }^{0}$ Standard error of the mean.

${ }^{1}$ Body weight at the $1^{\text {st }}$ day.

${ }^{2}$ Feed intake.

${ }^{3}$ Feed conversion ratio.

${ }^{4}$ Average weight gain.

${ }^{5}$ Daily weight gain.

concluded that providing a diet with $2,600 \mathrm{kcal}_{\mathrm{kg}}{ }^{-1}$ and $16 \% \mathrm{CP}$ would be ideal for optimal growth of poultry at starter phase.

For starter phase (8-21 days) no significant effects for FI (Table 4). However, for FCR, GAIN and ADG, significant difference $(\mathrm{P}<0.05)$ was observed.

The GAIN and ADG were on average $21 \mathrm{~g}$ and $1.5 \mathrm{~g} \mathrm{~d}^{-1}$ higher, respectively, for broilers fed with pre-starter, starter and growth phases. However, diets formulated with starter and growth 1 energy values did not differ statically from diets formulated with growth 2 and finishing phases.

The ADCn values determined in digestibility assay conducted by SCHNEIDERS et al. (2011) for each age, make the diets formulated to performance trial, since isocaloric for all ages included different energetic feedstuffs (corn and oil). Different inclusions contributed to energy metabolism, where, diets which have greater soybean oil inclusion (mainly in pre-start phase), were better used, significantly improving $(\mathrm{P}>0.05)$ the performance because lipids metabolism for energy generation is much faster than the carbohydrates metabolism (corn starch) for the same purpose.

The founded results are correlated to those discussed by MENDES et al. (2004) during 1-21 days. VASCONCELOS \& SANTOS (1997) and NASCIMENTO et al. (1998) also reported improvements in GAIN and FCR with increased dietary levels of energy; however, reported no differences for feed at this stage. However, MAIORKA et al. (1997) also evaluating different dietary energy levels for broilers reported no differences on performance variables within 7 to 21 days old.

Table 4 - Performance values determined with broiler chicken at starter phase ( 8 to 21 days).

\begin{tabular}{|c|c|c|c|c|c|c|c|}
\hline Characteristic & pre & Start & gro1 & gro2 & final & tab & $\mathrm{SEM}^{0}$ \\
\hline $\mathrm{BW}^{1}\left(\mathrm{~g} \mathrm{bird}^{-1}\right)$ & 171.300 & 171.230 & 171.330 & 171.360 & 171.330 & 171.720 & 0,064 \\
\hline $\mathrm{FI}^{2}\left(\mathrm{~g} \mathrm{bird}^{-1}\right)$ & 1006.030 & 1043.880 & 1042.940 & 1054.820 & 1044.810 & 1043.850 & 6,308 \\
\hline $\mathrm{FCR}^{3}\left(\mathrm{~g} \mathrm{~g}^{-1}\right)$ & $1.275^{\mathrm{a}}$ & $1.343^{\mathrm{b}}$ & $1.332^{\mathrm{b}}$ & $1.386^{\mathrm{b}}$ & $1.369^{\mathrm{b}}$ & $1.392^{\mathrm{b}}$ & 0,016 \\
\hline $\operatorname{GAIN}^{4}\left(\mathrm{~g} \mathrm{bird}^{-1}\right)$ & $789.470^{\mathrm{a}}$ & $777.170^{\mathrm{ab}}$ & $782.970^{\mathrm{ab}}$ & $761.440^{\mathrm{bc}}$ & $763.730^{\mathrm{bc}}$ & $750.560^{\mathrm{c}}$ & 5,487 \\
\hline $\mathrm{ADG}^{5}\left(\mathrm{~g} \mathrm{bird}^{-1}\right.$ day $\left.^{-1}\right)$ & $56.390^{\mathrm{a}}$ & $55.510^{\mathrm{ab}}$ & $55.920^{\mathrm{ab}}$ & $54.390^{\mathrm{bc}}$ & $54.550^{\mathrm{bc}}$ & $53.610^{\mathrm{c}}$ & 0,392 \\
\hline
\end{tabular}

\footnotetext{
*Means followed by the same letters in the same row. didnot differ by SNK test at 5\%.

${ }^{0}$ Standard error of the mean.

${ }^{1}$ Body weight at the $8^{\text {th }}$ day.

${ }^{2}$ Feed intake.

${ }^{3}$ Feed conversion ratio.

${ }^{4}$ Average weight gain.

${ }^{5}$ Daily weight gain.
} 


\section{CONCLUSION}

Diets formulated with energy values obtained on digestibility assays with different ages had significant effect on broiler performance at different ages. This demonstrated the great importance of considering the nutrients digestibility according to poultry's age.

\section{BIOETHICS AND BIOSSECURITY COMMITTEE APPROVAL}

We authors of the article entitled "Performance of broiler chickens at pre starter and starter phases using diets with different metabolizable energy values of ingredients, at different ages " declared, for all due purposes, that the project that gave rise to the present data was submitted for evaluation of the Ethics Committee of the "Universidade Estadual do Oeste do Paraná (UNIOESTE)", but we are aware of the content of the Brazilian resolutions of the Conselho Nacional de Controle de Experimentação Animal (CONCEA) <http://www.mct.gov.br/ index.php/content/view/310553.html $>$ if it involves animals.

Thus, the authors assume full responsibility for the present data and are available for possible questions, if they would be required by the competent authorities.

\section{ACKNOWLEDGEMENT}

To Conselho Nacional de Desenvolvimento Científico e Tecnológico $(\mathrm{CNPq})$ for financial support to perform this research.

\section{REFERENCES}

ALBUQUERQUE R. et al. Desempenho e perfil de produção de frangos de corte alimentados com dois níveis de energia na fase final e abatidos em três idades diferentes. In: REUNIÃO ANUAL DA SOCIEDADE BRASILEIRA DE ZOOTECNIA, 37., 2000, Viçosa, MG. Anais... Viçosa, MG: Sociedade Brasileira de Zootecnia, 2000. p.262.

BASTOS E.C.G. et al. Efeitos de níveis de energia da dieta e do sexo sobre o desempenho produtivo e rendimento de cortes nobres em frangos de corte. In: REUNIÃO ANUAL DA SOCIEDADE BRASILEIRA DE ZOOTECNIA, 35., 1998, Botucatu, SP. Anais.. Botucatu, SP: Sociedade Brasileira de Zootecnia, 1998. p.466-468.

DALE N.M.; FULLER H.L. Applicability of the true metabolizable energy system in practical feed formulation. Poultry Science, v.61, n.1, p.351-356, 1982.

HANUSHI S. et al. Effect of feeding different levels of energy and protein on peformance of Aseel breed of chicken during juvenile phase. Trop Animal Health and Production, v.44, n.7, p.1653-1658, 2012. Available from: <http://www.pubfacts.com/ detail/22453747> Accessed: May 18, 2012. doi: 10.1007/s11250012-0120-6. Epub 2012 Mar 29.

LARA L.J.C. et al. Influência da forma física da ração e da linhagem sobre o desempenho e rendimento de cortes de frangos de corte. Arquivo Brasileiro de Medicina Veterinária e Zootecnia, v.60, n.4, p.970-978, 2008.

LUCHESI J.B. Nutrição de frangos de corte. In: CONFERÊNCIA APINCO DE CIÊNCIA E TECNOLOGIA AVÍCOLA, 2000,
Campinas, SP. Anais... Campinas: Fundação APINCO de Ciência e Tecnologia Avícola, 2000. V.1, p.111-133.

MAIORKA A. et al. Efeito do nível energético da ração sobre o desempenho de frangos de corte de 1 7,7 a 14 e 14 a 21 dias de idade. In: CONFERÊNCIA APINCO DE CIÊNCIA E TECNOLOGIA AVÍCOLA, 1997, Campinas, SP. Anais... Campinas: Fundação APINCO de Ciência e Tecnologia Avícola, 1997. p.18

MENDES A.A. et al. Efeitos da energia da dieta sobre desempenho, rendimento de carcaça e gordura abdominal de frangos de corte. Revista Brasileira de Zootecnia, v.33, n.6, p.2300-2307, 2004. (Supl. 3).

MOREIRA J. et al. Efeito da densidade de criação e do nível de energia da dieta sobre o desempenho e rendimento de carcaça em frangos de corte. Revista Brasileira de Zootecnia, v.33, n.6, p.1506-1519, 2004.

NASCIMENTO A.H. et al. Valores de composição química e energética de alimentos para frangos de corte. Revista da Sociedade Brasileira de Zootecnia, v.27, n.3, p.579-583, 1998

ROSAA.P. et al. Desempenho e composição de carcaça de frangos submetidos a dietas com diferentes teores energéticos e níveis de gordura. In: REUNIÃO ANUAL DA SOCIEDADE BRASILEIRA DE ZOOTECNIA, 37., 2000, Viçosa, MG. Anais... Viçosa, MG: Sociedade Brasileira de Zootecnia, 2000. p.228.

ROSTAGNO H.S. et al. Tabelas brasileiras para aves e suínos: composição de alimentos e exigências nutricionais. Viçosa: UFV, Departamento de Zootecnia, 2005. 186p.

SAKOMURA, N.K.; ROSTAGNO, H.S. Métodos de pesquisa em nutrição de monogástricos. Jaboticabal: Funep, 2007. 283p.

SCHNEIDERS J.L. et al. Valores de energia metabolizável de diferentes alimentos de origem vegetal determinados com aves em diferentes idades. In: CONGRESSO BRASILEIRO DE ZOOTECNIA, 2011, 21., Maceió, AL. Anais... Maceió: UFAL: ABZ, 2011. CD-ROM. Zootec 2011.

SILVA J.H. et al. Energia e relações energia:proteína para frangos de corte de 22 a 42 dias de idade. In: REUNIÃO ANUAL DA SOCIEDADE BRASILEIRA DE ZOOTECNIA, 37., 2000, Viçosa, MG. Anais... Viçosa, MG: Sociedade Brasileira de Zootecnia, 2000. p.264.

VASCONCELOS R.Q.; SANTOS M.W. Efeito de níveis de energia e proteína da dieta sobre o desempenho de frangos de corte na fase inicial. In: REUNIÃO ANUAL DA SOCIEDADE BRASILEIRA DE ZOOTECNIA, 34., 1997, Juiz de Fora, MG. Anais... Juiz de Fora: Sociedade Brasileira de Zootecnia, 1997. V.4, p.6-8.

UNIVERSIDADE FEDERAL DE VIÇOSA (UFV). Manual de utilização do programa SAEG (Sistema para Análise Estatísticas e Genéticas). Viçosa, 1999. 59p.

VIANA C.F.A. et al. Influência de grupos genéticos e de níveis de energia sobre características de carcaça de frangos de corte. Revista Brasileira de Zootecnia [online], v.29, n.4, p.1067-1073, 2000. ISSN 1806-9290.

WATANABE K. et al. Efeito do nível de energia metabolizável da dieta sobre o metabolismo energético de frangos de corte. In: REUNIÃO ANUAL DA SOCIEDADE BRASILEIRA DE ZOOTECNIA, 38., 2001, Piracicaba, SP. Anais... Piracicaba: Sociedade Brasileira de Zootecnia, 2001. p.762. 